\title{
Osteogenic abilities of bone marrow stromal cells are not defective in patients with osteonecrosis
}

\author{
Jeong Joon Yoo • Won Seok Song • Kyung-Hoi Koo • \\ Kang Sup Yoon $\cdot$ Hee Joong Kim
}

Received: 16 December 2007 / Accepted: 3 January 2008 / Published online: 19 February 2008

(C) Springer-Verlag 2008

\begin{abstract}
We investigated whether the proliferation activities and osteogenic capacities of bone marrow stromal cells (BMSCs) are depressed in patients with osteonecrosis of the femoral head (ONFH). BMSCs were isolated from fresh bone marrow of the iliac crests of 54 donors and were differentiated into osteogenic lineage in vitro. The results of 27 consecutive patients with ONFH (16 idiopathic and 11 alcohol-induced) were compared with those of 27 patients with a nonnecrotic hip disorder. The proliferative activities of BMSCs in patients with ONFH were not found to be reduced and their osteogenic capacities (alkaline phosphatase activity and calcium deposition amount) were unaltered during in vitro differentiation. Results from patients with
\end{abstract}

$\overline{\text { Each author certifies that his or her institution has approved or waived }}$ approval for the human protocol for this investigation and that all investigations were conducted in conformity with ethical principles of research.

J. J. Yoo $\cdot$ K.-H. Koo · K. S. Yoon · H. J. Kim

Department of Orthopaedic Surgery,

Seoul National University College of Medicine,

Seoul, South Korea

\section{W. S. Song}

Department of Orthopaedic Surgery,

Korea Cancer Center Hospital,

Seoul, South Korea

\section{H. J. Kim}

Medical Research Center, Seoul National University,

Seoul, South Korea

\section{H. J. Kim $(\bowtie)$}

Department of Orthopaedic Surgery,

Seoul National University Hospital,

28 Yeongeon-dong, Jongno-gu,

110-744 Seoul, South Korea

e-mail: oskim@snu.ac.kr idiopathic or alcohol-induced ONFH were similar to those of matched patients with nonnecrotic disorder. These findings suggest that the osteogenic potentials of BMSCs are not defective in patients with ONFH.

Résumé Nous pensions que la prolifération et l'activité des cellules ostéogéniques de la moelle osseuse (BMSCs) étaient diminuées chez les patients qui présentaient une ostéonécrose de la tête fémorale (ONFH). Matériel et méthodes : Les BMSCs ont été isolées de la moelle fraiche, prélevées sur la crête iliaque de 54 donneurs et classées selon leur lignée ostéogénique. Les résultats de 27 patients avec ostéonécrose, 16 idopathiques et 11 d'origine éthylique ont été comparés à 27 patients ne présentant pas de nécrose de hanche. Résultat : l'activité des cellules de moelle osseuse chez les patients avec ostéonécrose ont été trouvées diminuées et leur capacité ostéogénique (activité phosphatase alkaline et déposition calcique) ne sont pas altérées lors de l'examen in vitro. Résultat : les patients présentant une ostéonécrose idiopathique ou éthylique ont une activité ostéogénique similaire comparée à celle des patients ne présentant pas de troubles d'origine nécrotique.

\section{Introduction}

Osteonecrosis of the femoral head (ONFH) is a disorder that mainly affects patients in their third to fifth decades, and one which generally progresses to collapse during the late stage of the disease [11, 15]. Although different pathophysiological mechanisms have been proposed to explain the pathogenesis of ONFH, such as fat emboli [7], microfracture of trabecular bone [8], compression of the blood vessels of the femoral head by hypertrophic marrow fat [22], retrograde embolisation of the marrow fat [17], 
coagulation and fibrinolytic disorder [1] and endothelial nitric oxide synthase (eNOS) gene deficiency [2], its precise cause is uncertain.

Human bone marrow stromal cells (hBMSCs) have the ability to self-renew and differentiate into several mesenchymal origin cells, such as osteoblasts, chondrocytes and adipocytes [14]. Moreover, hBMSCs may play important roles during the initial processes of fracture healing and skeletal repair [13].

Reports published recently suggest that BMSC numbers and osteogenic differentiation ability are depressed in patients with $\mathrm{ONFH}$, which, in turn, suggests that ONFH is a disease of bone cells and/or BMSCs $[5,6,9,20]$. In addition, several reports have indicated that BMSC isolation yield and osteogenic capacity are related to the risk factors of ONFH $[6,9]$. However, both patients with ONFH and patients without necrosis show similar levels of bone ingrowth into implants after arthroplasty [15, 24], and no report has indicated that autologous bone graft healing rates are reduced in patients with ONFH. Moreover, there is evidence that active new bone formation occurs in femoral heads around necrotic areas in most patients with ONFH [4]. These clinical findings suggest that the osteogenic potentials of bone-forming stem cells are not defective in patients with ONFH and that reductions in their activities is not a cause of ONFH.

We, therefore, asked whether patients with idiopathic or alcohol-induced ONFH have BMSCs with reduced proliferative activity and altered osteogenic capacities during in vitro osteogenic differentiation.

\section{Materials and methods}

The study population comprised 27 consecutive patients with idiopathic or alcohol-induced ONFH and 27 patients with nonnecrotic hip disorders with no history of heavy drinking. All patients were treated by total hip arthroplasty (THA). We excluded patients with a concurrent illness, systemic disease or a medical history that potentially affected bone metabolism, such as corticosteroid medication. No differences were observed between patients with ONFH and nonnecrotic patients in terms of age or gender (Table 1). All patients were fully informed of the study rationale, protocol and associated risks. The study was approved by our Institutional Review Board. The ONFH group included 18 men and nine women, with an overall mean age of 52.8 years (range, 23-78 years). ONFH was diagnosed radiographically or by magnetic resonance imaging (MRI). The idiopathic ONFH subgroup consisted of 16 patients (nine men, seven women) of mean age 54.1 years (range, 23-78 years), and the alcohol-induced ONFH subgroup comprised 11 patients (nine men, two women), with a mean age of 50.9 years (range, 3265 years). According to Steinberg et al.'s staging system [18], two ONFH patients were in Stage III, 12 were in Stage IV, nine in Stage V and four in Stage VI. The number of ONFH patients recruited was calculated based on a standardised effect size of 0.80 and power models with alpha and beta values of 0.05 and 0.20 , respectively.

For alcohol-induced ONFH to develop in adults, the total alcohol exposure threshold needs to be approximately $150 \mathrm{~L}$ of $100 \%$ ethanol, which corresponds to a consumption of $400 \mathrm{~mL}$ or more of absolute ethanol per week [23]. We determined the weekly alcohol consumption by summing the ethanol contents of beverage types consumed over a typical week. In all patients with alcohol-induced ONFH, the amount of alcohol consumed exceeded $400 \mathrm{~mL}$ weekly and the total alcohol consumption threshold level of $150 \mathrm{~L}$. In the alcohol-induced ONFH subgroup, five of the 11 patients had stopped drinking, four continued to consume alcohol but at reduced levels and two were still drinking heavily. The nonnecrotic hip disorder group included 17 men and 10 women aged 24 to 79 years (mean, 48.9 years). These disorders were primary coxarthrosis or coxarthrosis after hip dysplasia in 15 patients, sequelae of a hip joint infection in eight, fracture of the proximal femur in two and miscellaneous conditions in two.

We (JJY and HJK) aspirated fresh bone marrow from the iliac crests of donors just before starting THA. A 14-gauge needle was inserted into the iliac tuberosity adjacent to the anterior superior iliac spine by hand, and 6 to $7 \mathrm{~mL}$ of marrow was aspirated within 3 to $8 \mathrm{~s}$. The obturator and needle were then advanced by $10 \mathrm{~mm}$ twice to obtain two more aspirates through the same cortical hole. A total of $20 \mathrm{~mL}$ of marrow aspirate was collected per patient into a syringe containing 6,000 units of heparin. BMSCs were isolated from marrow aspirates using a modification of the procedure described by Pittenger et al. [14]. Briefly, marrow samples were washed with Dulbecco's phosphatebuffered saline (GibcoBRL, Gaithersburg, MD) and cells were recovered by centrifuging twice at $900 \mathrm{~g}$ for $10 \mathrm{~min}$. We then resuspended washed cells in Dulbecco's phosphate-buffered saline to a final density of $4 \times 10^{7}$ cells $/ \mathrm{mL}$, and $5-\mathrm{mL}$ aliquots were layered over $1.073 \mathrm{~g} / \mathrm{mL}$ Percoll solution (Pharmacia, Piscataway, NJ) in a $50-\mathrm{mL}$ conical tube and centrifuged at $1,100 \mathrm{~g}$ for $30 \mathrm{~min}$. Mononuclear cells were collected from interfaces and counted using a haemacytometer. Collected nucleated cells were resuspended in human MSC medium and plated in $150-\mathrm{mm}$ Petri dishes at $2 \times 10^{6}$ cells per dish in $30 \mathrm{~mL}$ of medium. Human MSC medium consisted of Dulbecco's Modified Eagles Medium-Low Glucose (GibcoBRL) supplemented with $10 \%$ foetal bovine serum (GibcoBRL), $25 \mathrm{U} / \mathrm{mL}$ of penicillin (GibcoBRL), $200 \mathrm{mM}$ of L-glutamine (Sigma, St Louis, MO) and $25 \mu \mathrm{g} / \mathrm{mL}$ of streptomycin (GibcoBRL). 
Table 1 Demographic data

\begin{tabular}{lllll}
\hline & \multicolumn{2}{l}{ ONFH $^{*}$} & $\begin{array}{l}\text { Nonnecrotic } \\
\text { disorder }\end{array}$ \\
\cline { 2 - 4 } & Idiopathic & $\begin{array}{l}\text { Alcohol- } \\
\text { induced }\end{array}$ & Total & \\
\hline Male:female & $9: 7$ & $9: 2$ & $18: 9$ & $17: 10$ \\
Mean age (years) & 54.1 & 50.9 & 52.8 & 48.9 \\
Age & $23-78$ & $32-65$ & $23-78$ & $24-79$ \\
distribution (years) & & & & \\
Steinberg stage & & & & \\
III & 1 & 1 & 2 & - \\
IV & 5 & 7 & 12 & - \\
V & 8 & 1 & 9 & - \\
VI & 2 & 2 & 4 & - \\
\hline
\end{tabular}

*ONFH=osteonecrosis of the femoral head

hBMSC cultures were maintained in $5 \% \mathrm{CO}_{2}$ at $37^{\circ} \mathrm{C}$ and the media were replaced at 24 and $72 \mathrm{~h}$ and then every 3 to 4 days. On day 20 after seeding, cells were harvested by adding a solution containing $0.05 \%$ trypsin $+0.53 \mathrm{mM}$ EDTA (GibcoBRL), counted and cryopreserved. These cells were designated passage 0 .

A single researcher conducted experiments during osteogenic differentiation using thawed aliquots of first passaged BMSCs isolated from all subjects. The cells were plated and cultured in osteogenic medium at $37^{\circ} \mathrm{C}$ in $5 \%$ $\mathrm{CO}_{2}$ for $24 \mathrm{~h}$; the media were replaced with fresh medium every 3 to 4 days. Dishes became fully confluent after recovery culture for 1 week. The cells were then detached with trypsin, replated at $3 \times 10^{4}$ cells per well in 24-well plates and evaluated for osteogenic differentiation. The osteogenic medium consisted of Dulbecco's Modified Eagles Medium-Low Glucose (GibcoBRL) supplemented with $10 \%$ foetal bovine serum (GibcoBRL), $200 \mathrm{mM}$ of Lglutamine (Sigma), $0.1 \mu \mathrm{M}$ of dexamethasone (Sigma), $50 \mu \mathrm{g} / \mathrm{mL}$ of ascorbic acid-2-phosphate (Sigma), $10 \mathrm{mM}$ of $\beta$-glycerophosphate (Sigma), $25 \mathrm{U} / \mathrm{mL}$ of penicillin (GibcoBRL) and $25 \mu \mathrm{g} / \mathrm{mL}$ of streptomycin (GibcoBRL). On days $1,4,7$ and 10 after commitment to the osteogenic lineage, we determined the cell numbers using a cell counting kit (CCK-8; Dojindo, Tokyo, Japan). Alkaline phosphatase (ALP) activity was measured on days 7 and 14 using an ALP diagnostic kit (\#245; Sigma) to evaluate the osteogenic differentiation. ALP activity was calculated after measuring the absorbance of the reaction product, $p$ nitrophenol, at $405 \mathrm{~nm}$ on a microplate reader. ALP activity was expressed in terms of $\mu \mathrm{mol} p$-nitrophenol/min per well. In addition, we measured the calcium content on days 14 and 21 using a calcium detection kit (\#587; Sigma) to assess the osteogenic mineralisation of BMSCs. Cell layers were washed three times with $\mathrm{Ca}^{2+}$ and $\mathrm{Mg}^{2+}$-free phosphate-buffered saline and then solubilised with $0.6 \mathrm{~N}$ $\mathrm{HCl}$. The samples from each well were then reacted with $o$ - cresol-phthalein-complexon and colourimetric reaction was read at $575 \mathrm{~nm}$. Absolute calcium concentrations were then read off a standard calcium curve, according to the manufacturer's instructions (Sigma). The calcium contents are expressed in micrograms per well. During osteogenic differentiation, all assays were conducted seven times.

The Mann-Whitney test was used to compare the proliferative activities and the osteogenic capacities of BMSCs of the ONFH and nonnecrotic hip disorder groups. To analyse the proliferation activities and osteogenic differentiation potentials with respect to the two risk factors (idiopathic and alcohol-induced ONFH) separately, we compared the results with those of a consecutive age- and gender-matched group of patients with nonnecrotic hip disorder. Analyses were performed using SPSS version 11.5 (SPSS Inc., Chicago, IL).

\section{Results}

Patients with idiopathic or alcohol-induced ONFH did not show a reduction in BMSC proliferative activities during in vitro osteogenic differentiation (Fig. 1). Similarly, we observed no differences in the proliferative activities when patients with idiopathic or alcohol-induced ONFH were compared separately with age- and gender-matched patients with nonnecrotic hip disorder (Fig. 1).

The BMSCs of ONFH patients show no alteration in osteogenic capacity in comparison to the BMSCs of nonnecrotic hip disorder patients during in vitro differentiation (Figs. 2 and 3). In addition, the BMSCs of patients with idiopathic or alcohol-induced ONFH were similar to those of nonnecrotic hip disorder patients with respect to their osteogenic activities. Moreover, ONFH patients and nonnecrotic hip disorder patients showed no difference in ALP activities on days 7 and $14 \quad(p=0.238$ and $p=0.066$, respectively), and the ALP activities of patients with

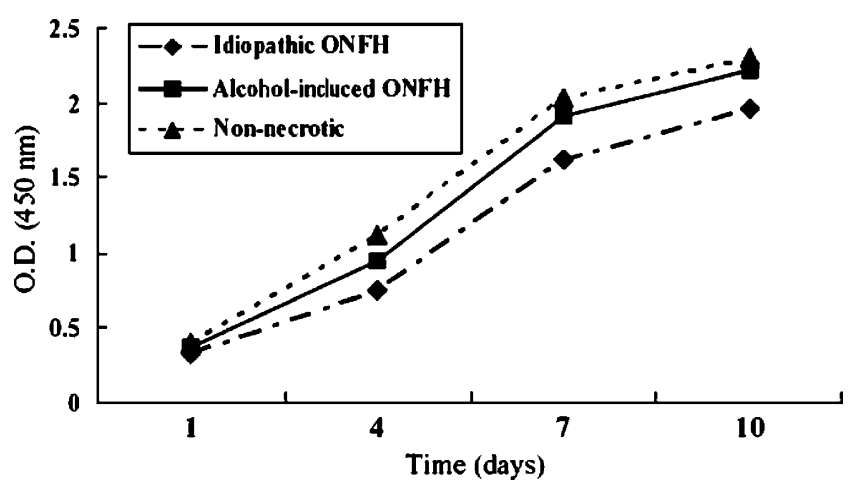

Fig. 1 The proliferative activities of human bone marrow stromal cells (hBMSCs) during osteogenic differentiation are presented with optical densities of CCK-8 assay. ONFH=osteonecrosis of the femoral head 
idiopathic, alcohol-induced ONFH and nonnecrotic hip disorder patients were not significantly different on days 7 or 14 (Fig. 2). The calcium content deposited by the BMSCs of patients with idiopathic ONFH and nonnecrotic hip disorder were similar on days 14 and $21(p=0.271$ and $p=$ 0.144 , respectively) and, furthermore, no differences were observed between the nonnecrotic hip disorder group and the idiopathic or alcohol-induced groups in this respect (Fig. 3).

\section{Discussion}

In this study, the data obtained demonstrate that the proliferative activities and osteogenic differentiation abilities of BMSCs isolated from the iliac crest in patients with idiopathic or alcohol-induced ONFH are not defective compared with those of patients with a nonnecrotic hip disorder. Furthermore, when patients with idiopathic or alcohol-induced ONFH were compared with age- and gender-matched nonnecrotic hip disorder patients, no significant differences were observed in terms of BMSC proliferation activities or osteogenic potentials.

However, our experimental design has its limitations. First, BMSCs activities were not measured when ONFH occurred, and most of our subjects had late-stage disease and showed joint destruction when the bone marrow was aspirated. Moreover, the time between disease occurrence and marrow cell harvest can permit the recovery of an abnormal bone marrow function. In particular, this recovery might have occurred in the five patients with alcoholinduced ONFH who had stopped drinking before study commencement. However, it is difficult to obtain bone marrow specimens from patients with earlier-stage $\mathrm{ONFH}$ because of the absence of subjective symptoms and ethical concerns. Moreover, several researchers have reported reduced BMSC osteogenic differentiation in ONFH patients

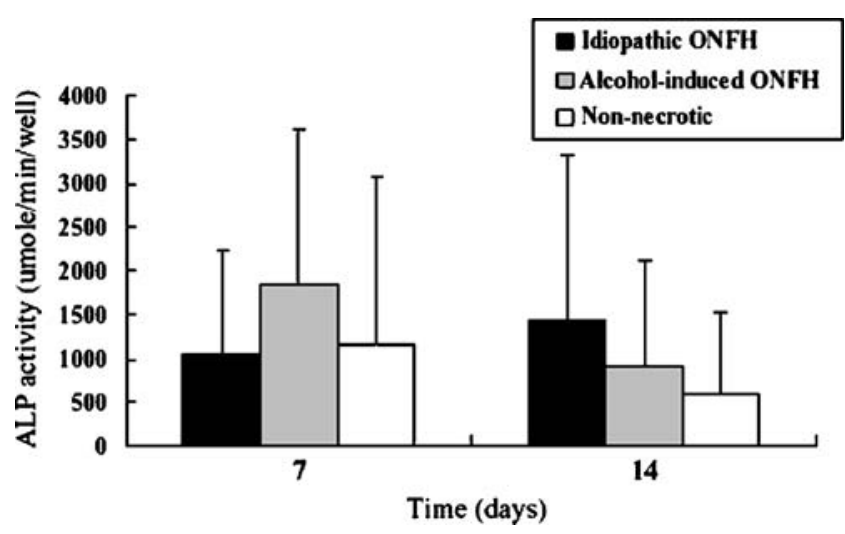

Fig. 2 Alkaline phosphatase (ALP) activity of hBMSCs during osteogenic differentiation is shown. No significant differences are observed between patients with ONFH and patients with nonnecrotic hip disorder

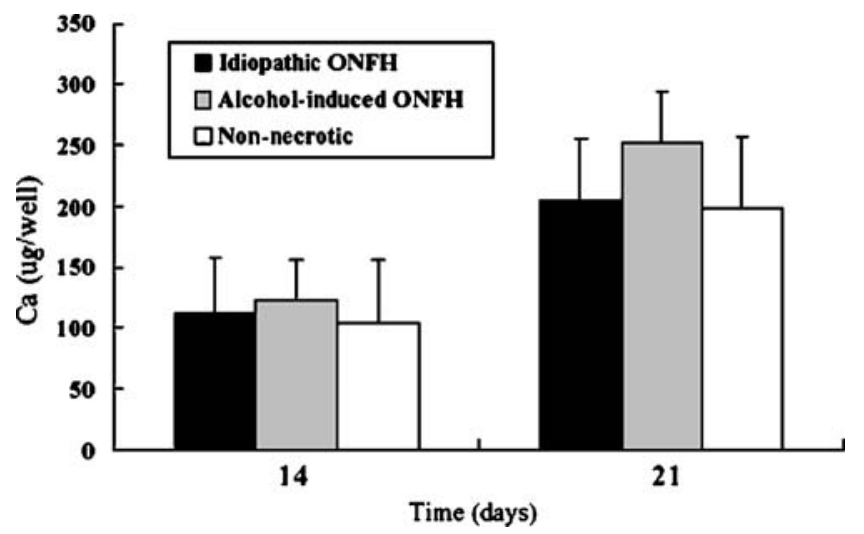

Fig. 3 Amount of calcium deposited by hBMSCs during osteogenic differentiation is shown. No significant difference is found between hBMSCs obtained from patients with ONFH and patients with nonnecrotic hip disorder

requiring hip replacement for late-stage disease $[9,20]$. In our study, BMSCs activities were not found to be significantly different in alcohol-induced ONFH patients who had stopped drinking and those that continued to consume alcohol (data not shown). Second, we acknowledge that the differentiation of BMSCs is highly dependent on in vivo and in vitro conditions, and, thus, our in vitro findings may not reflect the in vivo environment. Although we used the same passage cells from each group, freshly prepared cells may have better represented the in vivo condition. However, we also consider that interpersonal variances and potential experimental bias are likely to increase when assays are conducted separately using freshly prepared cells.

It remains controversial as to whether isolation yield and proliferation capacity are greatly dependent on donor age $[10,12,19]$, gender or on the presence of systemic disease [21]. We postulated that donor-specific variables can affect bone marrow cells, and, thus, to minimise the potentially confounding effects of these donor variables, we matched ONFH patients with nonnecrotic hip disorder patients for age and gender.

A small number of research groups have investigated bone marrow cell activities in patients with ONFH [3, 5, 6, $9,20]$. Hernigou and Beaujean [5] examined the activities of BMSCs from the iliac crest and reported that the number of CFU-Fs during isolation culture was lower in patients with idiopathic, alcohol-induced or steroid-induced ONFH than in the control group. They also demonstrated a reduction in the number of CFU-Fs in the upper end of the femur in patients with steroid-induced ONFH as compared with patients with sickle cell disease-related ONFH, a normal control group and patients with sickle cell disease but no ONFH [6]. However, interestingly, no major difference was observed between patients with sickle cell disease-related ONFH and a nonnecrotic control group 
[6]. Recently, Suh et al. [20] reported that BMSCs obtained from proximal femur marrow in patients with alcoholinduced ONFH had lower osteogenic differentiation ability and doubling time than cells obtained from patients with femoral neck fractures. However, there is a possibility that BMSCs in the control group were activated in response to these fractures. Moreover, Lee et al. [9] (in the same research group as Suh et al.) demonstrated that the osteogenic differentiation ability of BMSCs in patients with alcohol-related or idiopathic ONFH was lower than in patients with osteoarthritis. However, interestingly, they also found that BMSC osteogenic differentiation ability in patients with steroid-induced ONFH was unchanged. These results contradict Hernigou and Beaujean's findings, which showed reduced BMSC activity in the proximal femurs of patients with steroid-induced ONFH. However, it should be added that the evaluation parameters (osteogenic differentiation ability versus number of CFU-Fs), bone marrow aspiration techniques and cell isolation methods used differed [6].

For the same reasons, it is difficult to compare our results with those of other researchers. Nevertheless, we believe that previous studies are limited because they failed to document whether BMSC osteogenic capacities are depressed in patients with ONFH. Moreover, this former research was conducted using one assay only (CFU-F numbers) during the cell isolation process, and cellular behaviour in terms of osteogenic differentiation in culture were not evaluated $[5,6]$. In addition, in two studies, subjects were not matched for donor age and gender [9, 20], and the results were contradictory, i.e. a depressed osteogenic differentiation ability in patients with alcoholinduced or idiopathic ONFH, but not in those with steroidinduced ONFH [9].

In our study, we found substantial donor-dependent variations in terms of the osteogenic potential of BMSCs. Recently, variable ALP expression in human BMSCs was noted as a result of sampling method and cellular heterogeneity among the donor population [16]. Although not analysed, we believe that inherent bone marrow aspiration procedure errors, e.g. peripheral blood or fatty marrow contamination, contribute to these donor-dependent variations. However, to the best of our knowledge, no established guidelines have been issued on aspiration methods or on volumes for evaluating bone marrow cells. Moreover, we consider that inconsistencies in bone marrow aspiration procedures should be considered when interpreting marrow-derived stromal cell results.

Our data suggests that patients with ONFH have no remarkable abnormalities in the osteogenic potentials of their stem cells. Moreover, our results do not support the hypothesis that a general reduction in the osteogenic capacity of BMSCs causes ONFH.
Acknowledgements The authors thank Jeong Hyun Bang, B.S., for technical assistance with the biological assay.

This work was supported by a grant (no. 04-2006-088) from the Seoul National University Hospital Research Fund.

\section{References}

1. Asano T, Takahashi KA, Fujioka M, Inoue S, Ueshima K, Hirata T, Okamoto M, Satomi Y, Nishino H, Tanaka T, Hirota Y, Kubo T (2004) Relationship between postrenal transplant osteonecrosis of the femoral head and gene polymorphisms related to the coagulation and fibrinolytic systems in Japanese subjects. Transplantation 77:220-225

2. Calder JD, Buttery L, Revell PA, Pearse M, Polak JM (2004) Apoptosis - a significant cause of bone cell death in osteonecrosis of the femoral head. J Bone Joint Surg Br 86:1209-1213

3. Cui Q, Wang GJ, Balian G (1997) Steroid-induced adipogenesis in a pluripotential cell line from bone marrow. J Bone Joint Surg Am 79:1054-1063

4. Glimcher MJ, Kenzora JE (1979) The biology of osteonecrosis of the human femoral head and its clinical implications. III. Discussion of the etiology and genesis of the pathological sequelae; comments on treatment. Clin Orthop Relat Res 140:273-312

5. Hernigou P, Beaujean F (1997) Abnormalities in the bone marrow of the iliac crest in patients who have osteonecrosis secondary to corticosteroid therapy or alcohol abuse. J Bone Joint Surg Am 79: 1047-1053

6. Hernigou P, Beaujean F, Lambotte JC (1999) Decrease in the mesenchymal stem-cell pool in the proximal femur in corticosteroid-induced osteonecrosis. J Bone Joint Surg Br 81:349-355

7. Jones JP Jr (1985) Fat embolism and osteonecrosis. Orthop Clin North Am 16:595-633

8. Laurent J, Meunier P, Courpron P, Edouard C, Bernard J, Vignon G (1973) Research on the pathogenesis of aseptic necrosis of the femoral head. Evaluation of the constitutional bony factors in 35 cases of iliac crest biopsy. Nouv Presse Med 2:1755-1760

9. Lee JS, Lee JS, Roh HL, Kim CH, Jung JS, Suh KT (2006) Alterations in the differentiation ability of mesenchymal stem cells in patients with nontraumatic osteonecrosis of the femoral head: comparative analysis according to the risk factor. J Orthop Res 24:604-609

10. Leskelä HV, Risteli J, Niskanen S, Koivunen J, Ivaska KK, Lehenkari P (2003) Osteoblast recruitment from stem cells does not decrease by age at late adulthood. Biochem Biophys Res Commun 311:1008-1013

11. Mont MA, Hungerford DS (1995) Non-traumatic avascular necrosis of the femoral head. J Bone Joint Surg Am 77:459-474

12. Mueller SM, Glowacki J (2001) Age-related decline in the osteogenic potential of human bone marrow cells cultured in three-dimensional collagen sponges. J Cell Biochem 82:583-590

13. Niedźwiedzki T, Dabrowski Z, Miszta H, Pawlikowski M (1993) Bone healing after bone marrow stromal cell transplantation to the bone defect. Biomaterials 14:115-121

14. Pittenger MF, Mackay AM, Beck SC, Jaiswal RK, Douglas R, Mosca JD, Moorman MA, Simonetti DW, Craig S, Marshak DR (1999) Multilineage potential of adult human mesenchymal stem cells. Science 284:143-147

15. Schneider W, Knahr K (2004) Total hip replacement in younger patients: survival rate after avascular necrosis of the femoral head. Acta Orthop Scand 75:142-146

16. Siddappa R, Licht R, van Blitterswijk C, de Boer J (2007) Donor variation and loss of multipotency during in vitro expansion of human mesenchymal stem cells for bone tissue engineering. J Orthop Res 25:1029-1041 
17. Simkin PA, Downey DJ (1987) Hypothesis: retrograde embolization of marrow fat may cause osteonecrosis. J Rheumatol 14:870 872

18. Steinberg ME, Hayen GD, Steinberg DR (1995) A quantitative system for staging avascular necrosis. J Bone Joint Surg Br 77: 34-41

19. Stenderup K, Justesen J, Eriksen EF, Rattan SI, Kassem M (2001) Number and proliferative capacity of osteogenic stem cells are maintained during aging and in patients with osteoporosis. J Bone Miner Res 16:1120-1129

20. Suh KT, Kim SW, Roh HL, Youn MS, Jung JS (2005) Decreased osteogenic differentiation of mesenchymal stem cells in alcoholinduced osteonecrosis. Clin Orthop Relat Res 431:220-222
21. Suzuki Y, Kim KJ, Kotake S, Itoh T (2001) Stromal cell activity in bone marrow from the tibia and iliac crest of patients with rheumatoid arthritis. J Bone Miner Metab 19:56-60

22. Wang GJ, Sweet DE, Reger SI, Thompson RC (1977) Fat-cell changes as a mechanism of avascular necrosis of the femoral head in cortisone-treated rabbits. J Bone Joint Surg Am 59:729-735

23. Wang Y, Li Y, Mao K, Li J, Cui Q, Wang GJ (2003) Alcoholinduced adipogenesis in bone and marrow: a possible mechanism for osteonecrosis. Clin Orthop Relat Res 410:213-224

24. Xenakis TA, Beris AE, Malizos KK, Koukoubis T, Gelalis J, Soucacos PN (1997) Total hip arthroplasty for avascular necrosis and degenerative osteoarthritis of the hip. Clin Orthop Relat Res 341:62-68 